\title{
Infectious uveitis in immunodeficient HIV-negative patients: A retrospective study
}

\author{
Petra Svozilkova, Eva Rihova, Michaela Brichova, Andrea Havlikova, Aneta Klimova, Jarmila Heissigerova
}

\begin{abstract}
Aims. Cases of infectious uveitis in immunodeficient patients may present with atypical clinical features because the clinical course of disease is usually affected by the compromised immune system. Therefore, it is sometimes difficult to determine the correct diagnosis. The aim of this study was to evaluate a prevalence of immunodeficient HIV-negative individuals among patients with infectious uveitis and to assess diagnostic and therapeutic approaches.

Methods. A retrospective study.

Results. In years 2003-2017, we diagnosed 594 patients with infectious uveitis. In 35 of them, infectious uveitis occurred on the basis of immunodeficiency (malignancy, immunosuppressive therapy etc.). The most common infectious uveitis was cytomegalovirus retinitis, followed by acute retinal necrosis, herpetic anterior uveitis, endogenous fungal endophthalmitis, toxoplasmic retinochoroiditis, progressive outer retinal necrosis and syphilis. In indicated cases, intraocular fluid examination was a valuable diagnostic tool. After initiation of treatment, improvement of BCVA was observed in 13 eyes, stabilization in 13 eyes and deterioration in 11 eyes. In some patients who experienced adverse effects of medication, the recommended doses were decreased.

Conclusion. Our experience shows that patients with diagnoses of acute retinal necrosis or progressive outer retinal necrosis have a poor visual prognosis. The occurrence of cytomegalovirus retinitis signifies a very unfavorable survival prognosis in patients who underwent hematopoietic stem cell transplantation; the patients in our study died within 1 year from cytomegalovirus retinitis diagnosis.
\end{abstract}

Key words: herpetic retinal necrosis, cytomegalovirus retinitis, immunodeficiency, fungal endophthalmitis, toxoplasmic retinochoroiditis

Received: April 14, 2019; Revised: June 11, 2019; Accepted: July 22, 2019; Available online: August 21, 2019

https://doi.org/10.5507/bp.2019.036

(c) 2020 The Authors; https://creativecommons.org/licenses/by/4.0/

Department of Ophthalmology, First Faculty of Medicine, Charles University in Prague and General University Hospital in Prague, Czech Republic

Corresponding author: Petra Svozilkova, e-mail: petra.svozilkova@lf1.cuni.cz

\section{INTRODUCTION}

Infectious uveitis accounts for approximately $17-23 \%$ of all uveitis cases in developed countries. The causative agents differ throughout the world depending on geographic and demographic factors. According to anatomical classification, the most common type of uveitis in Western countries is the anterior form. In contrast, posterior uveitis is more frequent in Africa, Asia and South America. Among infectious agents, the most common are herpes viruses and Toxoplasma gondii ${ }^{1-5}$. Many etiological agents are ubiquitous, e.g. seroprevalence of cytomegalovirus (CMV) rises depending on age of patients from 60 to $90 \%$. Immunocompetent people have usually no or minimal symptoms during initial infection. However, immunodeficient patients (AIDS, malignancy, immunosuppressive treatment after organ transplantation or due to autoimmune disease) may develop systemic infectious diseases, carrying a significant risk of morbidity and mortality ${ }^{6}$.

Cases of infectious uveitis in immunodeficient patients may present with atypical clinical features because the clinical course of disease is usually affected by the compromised immune system. Therefore, it is sometimes dif- ficult to determine the correct diagnosis. Examination of intraocular fluid can be a valuable tool for confirming the causative agent. In addition, medications and their dosages should be adjusted depending on the therapeutic response and possible toxic adverse effects in immunodeficient patients.

The purpose of this retrospective study was to evaluate a prevalence of immunodeficient HIV-negative individuals in a group of patients with infectious uveitis and to assess diagnostic and therapeutic approaches in these patients.

\section{METHODS}

A retrospective study was performed in our Centre for Diagnosis and Treatment of Uveitis, First Faculty of Medicine, Charles University and General University Hospital in Prague. Thirty-five patients with immunodefiency and infectious uveitis out of 2794 patients with uveitis were included during the period of 2003-2017. All of them were HIV-negative. Diagnostic methods and the effect of treatment including the best-corrected visual acuity (BCVA) were evaluated.

Diagnostic procedures included medical history, 
Snellen BCVA, intraocular pressure measurement, slit lamp biomicroscopy, anterior segment and fundus examination. In indicated cases also visual field test, ultrasonography, optical coherence tomography and intraocular fluid examination were performed.

Therapeutic approaches included the initiation of antiinfectious agents in all patients, and pars plana vitrectomy in 14 patients ( 3 patients with CMV retinitis, 5 patients with acute retinal necrosis - ARN, 1 patient with progressive outer retinal necrosis - PORN, and 5 patients with fungal endophthalmitis).

We evaluated age of patients at time of presentation of infectious uveitis, unilateral or bilateral involvement, initial and final BCVA, effect of therapy and possible complications. As improvement or deterioration of BCVA 2 or more lines of Snellen optotypes were considered.

Treatment of underlying disease was managed by a specialist (hematooncologist, rheumatologist, nephrologist etc.).

\section{RESULTS}

During the period of 15 years, we monitored and treated 594 patients with infectious uveitis. In 35 of them (5.9\%), infectious uveitis occurred on basis of immunodeficiency (malignancy, immunosuppressive therapy after organ transplantation or due to autoimmune disease).

The most common infectious uveitis in our study was CMV retinitis (11 patients); one of these patients had CMV chronic retinal necrosis. ARN (herpes simplex; HSV or varicella zoster virus; VZV) was diagnosed in 7 patients, PORN in 1 patient, herpetic anterior uveitis in 6 patients, endogenous fungal endophthalmitis in 6 patients, toxoplasmic retinochoroiditis in 3 patients and syphilis in 1 patient (Table 1).

\section{CMV retinitis}

Among the patients with CMV retinitis were 8 men and 3 women. Nine of them had bilateral retinitis; the remaining 2 patients had unilateral ocular involvement. The age range was 18-58 years (median 44 years).

Five patients underwent hematopoietic stem cell transplantation (HSCT) due to hematological malignancy. The main cause was acute myeloid leukemia in $80 \%$ of cases. CMV retinitis occurred in 5-12 months after transplantation. Two patients had lymphoma (1 patient diffuse large B-cell lymphoma; DLBCL and 1 patient follicular lymphoma). One patient underwent liver transplantation and was on immunosuppressive medication. Three patients received immunosuppressive treatment because of systemic autoimmune disease (ulcerous colitis, ANCA-associated vasculitis or psoriatic arthritis). The 58-year-old women with immunosuppression due to psoriatic arthritis met the criteria for CMV chronic retinal necrosis.

The main symptom were visual field defects in 5 patients and decrease of visual acuity in 6 patients. In 10 out of 20 eyes, macula was unaffected; in 10 eyes were changes in macula (inflammatory infiltration, macular edema, epiretinal membrane, subretinal hemorrhage, exudative retinal detachment).

BCVA was evaluated in 9 patients; 2 women were treated by another ophthalmologist after confirmation of diagnosis at our clinic. The best initial BCVA was $6 / 6$ and the worst was counting fingers with light perception with correct projection of rays. The best final BCVA was $6 / 6$ and the worst was blindness.

Type and doses of virostatic treatment were consulted with a specialist (hematooncologist, rheumatologist, nephrologist etc.). Four patients were treated with virostatics for systemic CMV infection before the development of CMV retinitis - early after HSCT in 3 patients and after chemotherapy due to lymphoma in 1 patient.

Three patients with CMV retinitis were treated with intravenous ganciclovir; 2 patients received foscarnet. Three patients were treated with oral ganciclovir. One patient with systemic lupus erythematosus had indolent form of CMV retinitis and used no virostatic therapy.

In addition, three patients underwent pars plana vitrectomy due to vitreous opacities. Vitreous sample analysis confirmed CMV in two patients; one of them had CMV chronic retinal necrosis and the second patient had CMV retinitis and systemic follicular lymphoma. Vitreous cytological examination revealed some atypical lymphoid elements in second patient but immunophenotyping did not detect any B-lymphocytes. Third patient had a negative PCR result for CMV (Table 2). However, virostatic therapy was initially effective in this patient. Subsequently, vitreous opacitities and cystoid macular edema occurred. In this case, immune recovery uveitis was considered.

After initiation of effective treatment, the improvement of BCVA was observed in 4 eyes of 3 patients; the stabilization of BCVA was observed in 7 eyes of 7 patients. There was a decrease of BCVA in 6 eyes of 5 patients ( 2 eyes of patient with DLBCL, 4 eyes of 4 patients with complications - haemophthalmos, macular edema, exudative retinal detachment); (Table 3). Four patients, who underwent HSCT, died in 1, 4, 5 and 9 months after confirmation of CMV retinitis due to complications that were not related to virostatic treatment.

The follow-up duration was from 1 to 48 months (median 17 months).

\section{Acute retinal necrosis}

Among the patients with ARN were 4 men and 3 women. All of them had unilateral ocular involvement. The age range was 43-75 years (median 68 years).

Four patients had a history of malignancy (renal carcinoma, testicular carcinoma, systemic DLBCL, B-cell chronic lymphocytic leukemia); three patients were treated with immunosuppressive drugs because of autoimmune disease (ANCA-associated vasculitis, membranous glomerulonephritis, rheumatoid arthritis).

All patients were treated with aciclovir or valaciclovir; the doses were adjusted depending on the overall condition of the patients. In addition, six patients were treated with low doses of systemic corticosteroids due to dense vitreous opacities that are common in cases of ARN. A 
nephrologist recommended a reduction of doses of aciclovir in three patients with renal disease. The activity of inflammatory eye disease was under control in all patients.

Five patients underwent pars plana vitrectomy with laser barrage of necrotic retinal lesions and silicone oil instillation; four of them due to persistent vitreous opacities and one patient due to rhegmatogenous retinal detachment. PCR examination of vitreous samples confirmed VZV in 2 patients, HSV-1 in 1 patient, HSV-2 in 1 patient and 1 patient had negative result of PCR (Table 2) but vitreous cytologic examination supported the viral etiology of disease (as evidenced by an almost exclusive lymphocytic infiltration with cells in various stages of activation).

The best initial BCVA was 6/12 and the worst was counting fingers with light perception with correct projection of rays. In patients, who underwent pars plana vitrectomy, the best postoperative BCVA was $6 / 9$ and the worst $1 / 18$ with light perception with correct projection of rays. The best final BCVA was 6/18 and the worst was light perception with incorrect projection of rays. The improvement of BCVA was observed in 3 patients, the deterioration of BCVA in 4 patients (Table 3 ). The main causes for decrease of BCVA were a pale optic disc (4 patients), dense posterior capsule opacification (3 patients), macular edema (1 patient) and silicone oil keratopathy (1 patient).

The follow-up period was from 19 to 115 months (median 57 months).

Table 1. Etiological types of infectious uveitis in immunocompromised patients.

\begin{tabular}{lcccc}
\hline & $\mathrm{n}$ (patients) & $\%$ & Male-to-female ratio & Median age (years) \\
\hline Total & 35 & 100 & $22: 13$ & 54 \\
Herpetic uveitis & 25 & 71.4 & $17: 8$ & 58 \\
Cytomegalovirus retinitis & 11 & 31.4 & $8: 3$ & 44 \\
Acute retinal necrosis & 7 & 20.0 & $4: 3$ & 68 \\
Progressive outer retinal necrosis & 1 & 2.9 & $4: 0$ & 63 \\
Herpetic anterior uveitis & 6 & 17.1 & $3: 3$ & 54 \\
Fungal endophthalmitis & 6 & 17.1 & $1: 2$ & 73 \\
Toxoplasmic retinochoroiditis & 3 & 8.6 & $1: 0$ & 51 \\
Syphilis & 1 & 2.9 & & 39 \\
\hline
\end{tabular}

Table 2. Results of vitreous analyses in 14 patients indicated for pars plana vitrectomy.

\begin{tabular}{lcccc}
\hline Diagnosis & $\begin{array}{c}\mathrm{n} \\
\text { (patients) }\end{array}$ & $\begin{array}{c}\text { PCR } \\
\text { positive/negative }\end{array}$ & $\begin{array}{c}\text { Cultivation } \\
\text { positive/negative }\end{array}$ & Pathogen \\
\hline Cytomegalovirus retinitis & 3 & $2 / 1$ & - & CMV \\
Acute retinal necrosis & 5 & $4 / 1$ & - & VZV (2) \\
& & & HSV-1 (1) \\
& & & - & HSV-2(1) \\
Progressive outer retinal necrosis & 1 & $1 / 0$ & $3 / 2$ & VZV Candida albicans \\
Fungal endophthalmitis & 5 & - & & \\
\hline
\end{tabular}

PCR, polymerase chain reaction; CMV, cytomegalovirus; HSV-1, herpes simplex virus type 1; HSV-2, herpes simplex virus type 2; VZV, varicella zoster virus

Table 3. Results of BCVA in immunocompromised patients treated with anti-infectious drugs.

\begin{tabular}{llcccc}
\hline Diagnosis & Therapy & $\begin{array}{c}n \\
\text { (patients) }\end{array}$ & $\begin{array}{c}\text { Improvement of } \\
\text { BCVA } \\
\text { n (eyes) }\end{array}$ & $\begin{array}{c}\text { Stabilization of } \\
\text { BCVA } \\
\text { n (eyes) }\end{array}$ & $\begin{array}{c}\text { Deterioration of } \\
\text { BCVA } \\
n \text { (eyes) }\end{array}$ \\
\hline Cytomegalovirus retinitis & ganciclovir & 7 & 4 & 7 & 6 \\
foscarnet & 2 & & - & 4 \\
Acute retinal necrosis & aciclovir & 6 & 3 & - & 1 \\
Progressive outer retinal necrosis & valaciclovir & 1 & - & - \\
Herpetic anterior uveitis & aciclovir & 1 & 2 & 4 & - \\
Fungal endophthalmitis & fluconazole & 3 & 3 & - & - \\
Toxoplasmic retinochoroiditis & azithromycin & 2 & 1 & 1 & - \\
Syphilis & penicillin G & 1 & - & & \\
\hline
\end{tabular}

BCVA, best-corrected visual acuity. As improvement or deterioration of BCVA 2 or more lines of Snellen optotypes were considered. Patients who were referred to our clinic for confirmation of diagnosis only were excluded from BCVA evaluation. They were treated and followed up by another ophthalmologist. 


\section{Progressive outer retinal necrosis}

Unilateral PORN was diagnosed in a 63-year-old man. The patient underwent a heart transplantation three years ago, and nephrectomy due to Grawitz tumor two years ago. Since that time, he has been taking immunosuppressive drugs (tacrolimus, mycophenolate mofetil and corticosteroids). Moreover, the patient has type 2 diabetes mellitus on insulin medication.

Pars plana vitrectomy with retinectomy, laser barrage of necrotic retinal lesions, aciclovir lavage $(40 \mu \mathrm{g} / \mathrm{mL})$ and silicone oil instillation was performed 9 days after intravenous aciclovir initiation ( $1500 \mathrm{mg} /$ per day). PCR examination of vitreous samples confirmed VZV (Table 2).

The initial BCVA was $6 / 24$ in the affected eye. The postoperative BCVA was 6/12. Cataract surgery was performed in 11 months after pars plana vitrectomy. The final BCVA was 1/18 (Table 3). A dense posterior capsule opacification and a pale optic disc were present. A followup duration was 42 months.

\section{Herpetic anterior uveitis}

Herpetic anterior uveitis was diagnosed in 4 men and 2 women. All patients had unilateral ocular involvement. The age range was 26-74 years (median 54 years).

Three patients had malignancy (B-cell chronic lymphocytic leukemia, breast cancer, prostate cancer). Two of them were treated with chemotherapy. The patient with prostate cancer underwent radiotherapy. Other three patients were on immunosuppressive medication. Two of them underwent renal transplantation and were treated with tacrolimus, mycophenolate mofetil and corticosteroids. One patient had immunosuppression (azathioprine and corticosteroids) because of idiopathic bowel disease (ulcerous colitis).

All patients were treated with oral aciclovir; five of them with a common dose $2 \mathrm{~g}$ per day. In one patient, who underwent renal transplantation, a nephrologist recommended to initiate the treatment with lower dose of aciclovir ( $1.2 \mathrm{~g}$ per day) according to renal functions. The activity of anterior uveitis was under control in all patients. The 74-year-old woman with B-cell chronic lymphocytic leukemia was referred to our clinic only to confirm the diagnosis and to recommend an appropriate treatment. Aciclovir medication was conducted by another ophthalmologist.

The best initial BCVA was $6 / 6$ and the worst $6 / 36$. One patient had amblyopic eye and perceived hand movement and light perception with correct projection of rays. The best final BCVA was $6 / 6$ and the worst $6 / 9$ (counting fingers with light perception with correct projection of rays in amblyopic patient). The improved BCVA occurred in 2 patients, unchanged remained in 3 patients. No patient had deterioration of BCVA (Table 3 ). The follow-up period was from 29 to 139 months (median 64 months).

\section{Endogenous fungal endophthalmitis}

Three men and three women suffered from endogenous fungal endophthalmitis. Four patients had unilateral ocular involvement and two patients experienced bilateral endophthalmitis. The age range was $13-86$ years (median 73 years).

Three patients had malignancy (colorectal cancer, prostate cancer and multiple myeloma). Three patients had type 2 diabetes mellitus (two patients with malignancy and one patient with recent urinary infection treated with intravenous antibiotics). Two young patients had idiopathic bowel disease (ulcerous colitis, Crohn's disease). Both were treated with combination of corticosteroids and azathioprine.

Five patients ( 7 eyes) underwent pars plana vitrectomy with intravitreal amphotericin B. Candida albicans was detected in vitreous of three patients; two patients had negative results (Table 2). All patients were treated with fluconazole (200-400 mg per day). Two of these patients were referred to our clinic to confirm the diagnosis and to recommend effective therapy. Then they were in the care of a local ophthalmologist. The remaining 3 patients (4 eyes) had the best initial BCVA 6/12 and the worst counting fingers with light perception with correct projection of rays. The best final BCVA was 6/6 and the worst $6 / 9$. The improved BCVA occurred in all 3 patients ( 4 eyes); (Table 3).

The 73-year-old man with multiple myeloma underwent enucleation because of painful blind eye. The histological finding confirmed massive purulent inflammation with abscess in the vitreous and phlegmonous spread of inflammation in the retina and choroidea with the affection of the ciliary body. Fungal hyphae and spores were detected.

The follow-up period was from 11 to 32 months (median 19 months).

\section{Toxoplasmic retinochoroiditis}

Unilateral toxoplasmic retinochoroiditis was diagnosed in 1 man and 2 women. In two patients, it was a primary infection. A recurrence was identified in one patient. The age range was 38-61 years (median 51 years).

All three patients suffered from malignancy and underwent chemotherapy (ovarian cancer, colorectal cancer, chronic myeloid leukemia with HSCT). In addition, one patient had type 2 diabetes mellitus.

In all three patients, treatment with azithromycin ( 500 $\mathrm{mg}$ on the first day and then $250 \mathrm{mg}$ daily for 6-8 weeks) was initiated. One woman experienced a progression of the ocular finding despite of therapy. Furthermore, in this patient the signs of acute systemic infection were detected in blood samples. We referred this woman to the Infectious Disease Clinic where treatment with pyrimethamine, sulfadiazine and corticosteroids was started. The therapy was effective.

In two patients, the initial BCVA was $6 / 12$, resp. 6/9. The final BCVA was $6 / 6$ in both (Table 3 ). The 38 -year-old man was referred to our clinic for consultation. Follow-up was provided by a local ophthalmologist. The follow-up period in remaining 2 patients was 3 , resp. 57 months.

\section{Syphilis}

Unilateral mild optic disc edema and macular edema was observed in a 39-year-old man. The patient has been 
treated for 9 months by rheumatologist with azathioprine and corticosteroids for suspected Behcet's disease. The patient suffered from oral and genital aphthous ulcers. We suspected syphilis, so we indicated blood tests that confirmed the diagnosis. Penicillin therapy was initiated in cooperation with dermatovenerologist. The initial BCVA was 6/9; the final BCVA was 6/6 (Table 3). A follow-up duration was 12 months.

\section{DISCUSSION}

Larger series of immunodeficient HIV-negative patients with infectious uveitis of various etiology were rarely mentioned in publications. Hence, we retrospectively evaluated 35 patients with decreased immune status and active infectious uveitis.

In the past years, CMV retinitis was the main cause of decreased visual acuity in patients with AIDS (ref. ${ }^{7-}$ $\left.{ }^{9}\right)$. Since the introduction of highly active antiretroviral therapy (HAART), the proportion of CMV retinitis in this group of patients has been decreasing. HAART reduced the incidence of CMV retinitis by $80 \%$ (ref. ${ }^{10}$ ). Compared to that the incidence of CMV retinitis in HIV-negative immunodeficient patients may be increasing. However, this may be due to increasing numbers of patients with transplantation. The main risk group are patients after HSCT (ref. ${ }^{11}$ ). In our patients, CMV retinitis developed within 1 year after HSCT or organ (liver) transplantation. Similar results published Eid et al. 2008 and Kuo et al. 2004 (ref. ${ }^{12,13}$ ). In patients, who are treated with immunosuppression due to autoimmune disease, is the interval longer. Kuo et al. observed CMV retinitis in 8.5-21 months after initiation of immunosuppressive therapy ${ }^{13}$. In our three patients, the interval was 2, 4 and 15 years.

After initiation of virostatic treatment, we observed the improvement of BCVA in 4 eyes of 3 patients $(21 \%)$ and the stabilization of BCVA in 7 eyes of 7 patients (37\%). Eid et al. observed the improvement of BCVA in 4 eyes of 4 patients (28.5\%) and the stabilization of BCVA in 4 eyes of 4 patients (28.5\%) $\left(\right.$ ref. $\left.^{12}\right)$. The decrease of BCVA in 6 eyes of 5 our patients (32\%) was caused by complications (optic disc atrophy, macular edema, exudative retinal detachment, haemophthalmos).

Rhegmatogenous retinal detachment, a frequent complication of CMV retinitis, did not occur in our patients. However, some studies reported a manifestation of this complication. Eid et al. have experienced rhegmatogenous retinal detachment in 1 eye of 1 patient (out of 14 eyes of 9 patients) (ref. ${ }^{12}$ ). Kuo et al. observed this complication in 2 eyes of 2 patients (out of 30 eyes of 18 patients) between $1^{\text {st }}$ and $7^{\text {th }}$ month since the diagnosis determination. Two other patients in the same study had rhegmatogenous retinal detachment during their first visit ${ }^{13}$.

Four patients with CMV retinitis (36\%) died in 1, 4, 5 and 9 months after confirmation of diagnosis. All of them underwent HSCT. Kuo et al. reported deaths in 10 out of 18 patients (56\%) and mean survival time was 11 months after determining the diagnosis of CMV retinitis ${ }^{13}$.
In elderly patients, with a natural decline of activity of immune system, CMV retinitis appears to have an increased association with retinal arteriolar occlusions ${ }^{6,14,15}$. Some authors have found that in patients with diabetes mellitus or on noncytotoxic doses of immunosuppression due to autoimmune diseases, CMV retinitis can present with typical, slowly progressive, peripheral granular retinitis, and an atypical panretinal occlusive vasculitis, mimicking ARN (ref. ${ }^{6,16}$ ). Similar findings were seen in our 58-year-old woman with CMV chronic retinal necrosis, who used immunosuppression because of psoriatic arthritis. In addition, our patient had some opacities in vitreous, commonly seen in cases of chronic retinal necrosis, similarly to patients with ARN. Nevertheless, in cases of HSV or VZV ARN, vitreous haze is usually dense.

It is important to keep in mind that CMV retinitis is a part of systemic infection with implications for the second eye and other organs. This is the reason for initiation of virostatic therapy by systemic means, with or without concurrent intravitreal treatment ${ }^{6}$.

PORN and ARN have been described to represent two antipoles of the spectrum of herpetic necrotizing retinopathies. PORN typically affects immunodeficient patients. Compared to that, ARN predominantly affects immunocompetent people but less frequently can also develop in slightly immunocompromised patients ${ }^{17}$. Diagnosis of ARN is based on clinical findings alone using the American Uveitis Society's criteria ${ }^{18}$. The main risk of herpetic necrotizing retinopathies is bilateral involvement. In almost one-third of patients the second eye becomes involved, usually within 1-6 weeks ${ }^{19}$. In immunodeficient patients, the risk may be higher, especially in cases with poor tolerance of virostatics. All our patients had unilateral ocular involvement and the disease was under control on aciclovir or valaciclovir medication. Five patients underwent pars plana vitrectomy with transient improvement of BCVA. In the following period, the deterioration of BCVA was observed in 4 patients mainly due to optic disc atrophy and/or posterior capsule opacification. Optic disc swelling with subsequent atrophy is one of the main causes of BCVA decrease in patients with necrotizing herpetic retinopathies ${ }^{20,21}$.

Another type of infectious uveitis, which may occur in immunocompromised people, is endogenous fungal endophthalmitis. The main risk group are patients with malignancy, diabetes mellitus, on long-term immunosuppression or antibiotics, with indwelling intravenous catheters, or intravenous drug users. Early pars plana vitrectomy with intravitreal antifungal drugs is indicated in these patients. This surgical procedure enables to obtain vitreous samples for examination and usually leads to improvement of BCVA (ref. ${ }^{22}$ ). Five of our patients underwent pars plana vitrectomy with intravitreal administration of amphotericin B and were treated with oral fluconazole. The therapy was effective and resulted in improvement of BCVA. One patient primary underwent enucleation due to painful blind bulbus. The histological examination confirmed fungal endophthalmitis.

Furthermore, herpetic anterior uveitis, toxoplasmic 
retinochoroiditis and syphilis can affect both - immunodeficient or immunocompetent people.

All our patients with herpetic anterior uveitis had unilateral involvement. Treatment with oral aciclovir was effective and led to final BCVA between 6/6 to 6/9 (except one amblyopic patient). In patients who have only anterior uveitis without corneal scarring, visual prognosis is favorable 23,24 .

Toxoplasmic retinochoroiditis was diagnosed through clinical grounds alone in our patients. Generally, blood tests for detection of toxoplasma antibodies of acute phase (IgA, IgM) have only supporting significance and may be even negative in cases of isolated eye disease. The seroprevalence $(\mathrm{IgG})$ for Toxoplasma gondii is high in western Europe (41-75\%) whereas in Vietnam, China and Thailand it is low ${ }^{1,25-27}$. Causes for these regional differences are unknown; various factors such as diet, hygiene, infection of the environment by cyst and differences in pathogenicity of the regional strains might be involved ${ }^{1}$. Aqueous humor analysis may be helpful, especially if there are difficulties in determining the diagnosis. Westeneng et al. detected Toxoplasma gondii in aqueous humor in 1 out of 21 AIDS patients and in 10 out of 22 non-AIDS immunocompromised patients. They confirmed ocular toxoplasmosis in $45.5 \%$ of non-AIDS immunocompromised patients ${ }^{28}$. It was the most common infectious uveitis in this group of patients. In contrast, toxoplasmic retinochoroiditis was diagnosed in only $8.6 \%$ of cases in our study.

Serological testing is crucial for determination of syphilis. Syphilis is considered as "the great imitator" as it has many different manifestations and thus mimics many diseases. In addition, syphilis can be presented as any form of uveitis. Westeneng et al. revealed a strong correlation between having AIDS and ocular syphilis (6/21 in AIDS patients vs $0 / 22$ in non-AIDS patients) (ref. ${ }^{28}$ ). In our non-AIDS study, syphilis was confirmed in one patient. Ocular manifestation was mild optic disc swelling and discrete macular edema. Therapy was initiated immediately with consequent improvement of BCVA. The delay in treatment with penicillin can lead to long-standing optic neuropathy and cystoid macular edema, which is associated with poor BCVA $\left(\right.$ ref. $^{29}$ ).

In indicated cases, intraocular fluid examination is a valuable tool for determining the proper diagnosis through confirming the causative agent. Vitreous sampling allows multiple analyses due to higher volume of liquid compared to aqueous tapping. On the other hand, anterior chamber tapping is easy to perform, quick, and safe method ${ }^{30}$. PCR methods are widely used for detection of herpes viruses whereas analyses of intraocular antibody production play a decisive role in the diagnosis of ocular toxoplasmosis ${ }^{28}$. Bacterial or fungal intraocular inflammation is mainly confirmed by microscopic examination and cultivation of intraocular fluid samples. In our patients, pars plana vitrectomy was performed in 16 eyes ( 14 patients). Positive results of examination of vitreous samples were achieved in 11 patients $(78.6 \%)$.

The important key to the diagnoses of CMV retinitis, PORN and endogenous infectious endophthalmitis, is the history of immunodeficiency. Whereas other infectious uveitides, such herpetic anterior uveitis, ARN, toxoplasmic retinochoroiditis or syphilis may affect both - immunocompetent and immunocompromised people. The typical clinical features of uveitis can be altered due to immunodeficiency. Moreover, the therapy might not be effective in all cases. In many of our patients, type and dosages of medication were adjusted according to clinical response and possible toxic adverse effects. Therefore, interdisciplinary cooperation is crucial to appropriate care of our patients.

\section{CONCLUSION}

Among immunodeficient HIV-negative patients in our centre, the most common infectious uveitis was cytomegalovirus retinitis, followed by acute retinal necrosis, herpetic anterior uveitis, endogenous fungal endophthalmitis, toxoplasmic retinochoroiditis, progressive outer retinal necrosis and syphilis. Our experience shows that patients with a diagnosis of ARN or PORN have a poor visual prognosis. The occurrence of CMV retinitis signifies a very unfavorable survival prognosis in patients who have undergone HSCT; the patients in our study died within 1 year from CMV retinitisdiagnosis.

\section{ABBREVIATIONS}

ARN, Acute retinal necrosis; BCVA, Best-corrected visual acuity; CMV, Cytomegalovirus; DLBCL, Diffuse large B-cell lymphoma; HAART, Highly active antiretroviral therapy; HSCT, Hematopoietic stem cell transplantation; HSV, Herpes simplex virus; PCR, Polymerase chain reaction; PORN, Progressive outer retinal necrosis; VZV, Varicella zoster virus.

Acknowledgements: This work was supported by the Research Project of Charles University PROGRES Q25/ LF1/2. The authors thank our Vitreoretinal Department for performing pars plana vitrectomy in selected patients. They would also like to thank the Institute of Medical Biochemistry and Laboratory Diagnostics, First Faculty of Medicine, Charles University and General University Hospital in Prague for blood tests and intraocular fluid examination.

Author contributions: PS, ER: designed the study and data collection tools; PS: prepared and revised the manuscript; PS, ER, MB, AH, AK, JH: performed the examination and contributed to the discussion and content of the report. All authors read and approved the final manuscript. Conflict of interest statement: The authors state that there are no conflicts of interest regarding the publication of this article.

\section{REFERENCES}

1. Kongyai N, Pathanapitoon K, Sirirungsi W, Kunavisarut P, de GrootMijnes JD, Rothova A. Infectious causes of posterior uveitis and panuveitis in Thailand. Jpn J Ophthalmol 2012;56:390-5. 
2. Wakefield D, Chang JH. Epidemiology of uveitis. Int Ophthalmol Clin 2005;45:1-13.

3. Rathinam SR, Namperumalsamy P. Global variation and pattern changes in epidemiology of uveitis. Indian J Ophthalmol 2007;55:173-83.

4. London NJ, Rathinam SR, Cunningham ET Jr. The epidemiology of uveitis in developing countries. Int Ophthalmol Clin 2010;50:1-17.

5. Tsirouki T, Dastiridou A, Symeonidis C, Tounakaki O, Brazitikou I, Kalogeropoulos C, Androudi S. A Focus on the Epidemiology of Uveitis. Ocul Immunol Inflamm 2016:28:1-15.

6. Butler NJ, Thorne JE. Update on Diagnosis and Treatment of CMV Retinitis. Retinal Physician 2014;11:46-53.

7. Freeman WR, Friedberg DN, Berry C, Quiceno Jl, Behette M, Fullerton SC, Munguia D. Risk factors for development of rhegmatogenous retinal detachment in patients with cytomegalovirus retinitis. Am J Ophthalmol 1993;116:713-20.

8. Jabs DA. Ocular manifestations of HIV infection. Trans Am Ophthalmol Soc 1995;93:623-83.

9. Kempen JH, Jabs DA, Wilson LA, Dunn JP, West SK, Tonascia JA Risk of vision loss in patients with cytomegalovirus retinitis and the acquired immune deficiency syndrome. Arch Ophthalmol 2003;121:466-76.

10. Goldberg DE, Smithen LM, Angelilli A, Freeman WR. HIV-associated retinopathy in the HAART era. Retina 2005;25:633-49.

11. Xhaard A, Robin M, Scieux C, de Latour RP, Deplus S, Mazeron MC, Devergie A, Espérou H, Rocha V, Gluckman E, Ribaud P, Socié G. Increased incidence of cytomegalovirus retinitis after allogenic hematopoietic stem cell transplantation. Transplantation 2007;83:80-3.

12. Eid AJ, Bakri SJ, Kijipittayarit S, Razonable RR. Clinical features and outcomes of cytomegalovirus retinitis after transplantation. Transpl Infec Dis 2008;10:13-18.

13. Kuo IC, Kempen JH, Dunn JP, Vogelsang G, Jabs DA. Clinical charac teristics and outcomes of cytomegalovirus retinitis in persons without human immunodeficiency virus infection. Am J Ophthalmol 2004;138:338-46.

14. Davis JL, Haft P, Hartley K. Retinal arteriolar occlusions due to cytomegalovirus retinitis in elderly patients without HIV. J Ophthalmic Inflamm Infect 2013;3:17.

15. Gupta S, Vemulakonda GA, Suhler EB, Yeh S, Albini TA, Mandelcorn E, Flaxel CJ. Cytomegalovirus retinitis in the absence of AIDS. Can J Ophthalmol 2013:48:126-9.

16. Schneider EW, Elner SG, van Kuijk FJ, Goldberg N, Lieberman RM, Eliott D, Johnson MW. Chronic retinal necrosis: cytomegalovirus necrotizing retinitis associated with panretinal vasculopathy in non-HIV patients. Retina 2013;33:1791-9.
17. Guex-Crosier Y, Rochat C, Herbort CP. Necrotizing herpetic retinopathies. A spectrum of herpes virus-induced diseases determined by the immune state of the host. Ocul Immunol Inflamm 1997;5:259-65.

18. Holland GN. Standard diagnostic criteria for the ARN syndrome. Executive Committee of the American Uveitis Society. Am J Ophthalmol 1994;117:663-7.

19. Nussenblatt $R$, Whitcup $S$. Uveitis: Fundamentals and clinical practice. Fourth Edition. Philadelphia: Mosby Elsevier; 2010.

20. Tran TH, Bodaghi B, Rozenberg F, Cassoux N, Fardeau C, LeHoang P. Viral cause and management of necrotizing herpetic retinopathies. J Fr Ophtalmol 2004;27:223-36.

21. Oe C, Hiraoka M, Tanaka S, Ohguro H. Acute Retinal Necrosis Associated with Epstein-Barr Virus in a Patient Undergoing Immunosuppressive Therapy. Case Rep Ophthalmol 2016;7:195-201.

22. Behera UC, Budhwani M, Das T, Basu S, Padhi TR, Barik MR, Sharma S. Role of early vitrectomy in the treatment of fungal endophthalmitis. Retina 2018:38:1385-92.

23. Kardeş E, Bozkurt K, Sezgin Akçay Bï, Ünlü C, Aydoğan Gezginaslan T, Ergin A. Clinical Features and Prognosis of Herpetic Anterior Uveitis. Turk J Ophthalmol 2016;46:109-13.

24. Tugal-Tutkun I, Otük-Yasar B, Altinkurt E. Clinical features and prognosis of herpetic anterior uveitis: a retrospective study of 111 cases. Int Ophthalmol 2010:30:559-65.

25. Sirirungsi W, Pathanapitoon K, Kongyai N, Weersink A, de GrootMijnes JD, Leechanachai P, Ausayakhun S, Rothova A. Infectious uveitis in Thailand: serologic analyses and clinical features. Ocul Immunol Inflamm 2009;17:17-22.

26. Li S1, Cui L, Zhao J, Dai P, Zong S, Zuo W, Chen C, Jin H, Gao H, Liu O. Seroprevalence of Toxoplasma gondii infection in female sterility patients in China. J Parasitol 2011;97:529-30.

27. Udonsom R, Lekkla A, Chung PT, Cam PD, Sukthana Y. Seroprevalence of Toxoplasma gondii antibody in Vietnamese villagers. Southeast Asian J Trop Med Public Health 2008;39:14-18.

28. Westeneng AC, Rothova A, de Boer JH, de Groot-Mijnes JD. Infectious uveitis in immunocompromised patients and the diagnostic value of polymerase chain reaction and Goldmann-Witmer coefficient in aqueous analysis. Am J Ophthalmol 2007;144:781-5.

29. Zhu J, Jiang Y, Shi Y, Zheng B, Xu Z, Jia W. Clinical manifestations and treatment outcomes of syphilitic uveitis in HIV-negative patients in China: A retrospective case study. Medicine (Baltimore) 2017:96:e8376. doi: 10.1097/MD.0000000000008376.

30. Chronopoulos A, Roquelaure D, Souteyrand G, Seebach JD, Schutz JS, Thumann G. Aqueous humor polymerase chain reaction in uveitis - utility and safety. BMC Ophthalmol 2016; 16:189. 